Zbl Arbeitsmed $2021 \cdot 71: 157-166$ https://doi.org/10.1007/s40664-021-00422-0 Eingegangen: 2. September 2020

Angenommen: 26. Januar 2021

Online publiziert: 10. März 2021

c c Der/die Autor(en) 2021

Daniela Borchart · Jean-Baptist du Prel · Hans Martin Hasselhorn

Fachgebiet Arbeitswissenschaft, Bergische Universität Wuppertal, Wuppertal, Deutschland

\title{
Effekte gesundheitsrelevanter Verhaltensweisen auf die subjektive Erwerbsperspektive älterer Beschäftigter in Deutschland
}

Verbleib im Arbeitsleben erscheint vor diesem Hintergrund wesentlich.

Als Indikator für die Dauer der Erwerbsteilhabe wird die subjektive Erwerbsperspektive (EP) angesehen, $\mathrm{zu}$ der als zentrale Komponente die eigene Bewertung, wie lange man noch arbeiten kann, zählt [23, 43]. Das zugrundeliegende Konzept dieser Bewertung stellt nach Hasselhorn und Ebener [14] die Arbeitsfähigkeit dar. Eine schlechte Gesundheit wie auch physische und mentale Arbeitsbelastungen gehen mit der Intention, frühzeitig das Erwerbsleben zu verlassen, einher [37]. Frühere Untersuchungen zeigten, dass die Bewertung des (noch arbeiten) Könnens mit der physischen und mentalen Gesundheit assoziiert ist $[12,23]$.

Die Gesundheit ist einer der am meisten untersuchten Einflussfaktoren für die Dauer der Erwerbsteilhabe [38]. Beschäftigte mit schlechter Gesundheit steigen im Mittel früher aus dem Erwerbsleben aus als jene mit guter Gesundheit [38, 39]. Auf individueller Ebene kann ein gesundheitsbewusster Lebensstil die Gesundheit in jedem Alter begünstigen. Frühere Studien zeigen, dass Verhaltensweisen wie ein normales Körpergewicht, Nichtrauchen und ein ausreichendes Maß an körperlicher Aktivität, die Gesundheit im mittleren Erwachsenenalter positiv beeinflussen können und das Morbiditäts- und Mortalitätsrisiko langfristig minimieren [3, 35, 36]. Allerdings ist die Mehrheit der älteren Beschäftigten in Deutschland zu wenig körperlich aktiv, übergewichtig, und gut ein Viertel von ihnen raucht [6, 9, 31, 44].

Gesundheitsbezogene Verhaltensweisen (GVh) werden durch individuelle (z.B. Geschlecht, Bildung) und kontextuale (z. B. Arbeitsbedingungen) Faktoren determiniert. So haben z. B. Männer ungünstigere Ernährungsgewohnheiten und sind häufiger übergewichtig als Frauen [27]. Eine geringere berufliche Qualifikation und eine manuelle Arbeitstätigkeit können früheren Studien nach häufiger mit körperlicher Inaktivität in der Freizeit, Adipositas und Rauchen einhergehen $[5,28]$. Andere Studien zeigten, dass diese Berufsgruppen in ihren Arbeiten häufiger mit physischen und psychosozialen Belastungen (z. B. Heben und Tragen schwerer Lasten, geringere Entwicklungsmöglichkeiten) konfrontiert sind [7] und ein höheres Risiko für eine Frühverrentung aufgrund verminderter Erwerbsfähigkeit haben [17, 21, 33, 40].

DerZusammenhangvon GVhund der EP älterer Beschäftigter wurde nach unserem Wissensstand bislang nicht erforscht. Bisherige Studienergebnisse zum Zusammenhang der Erwerbsteilhabe und GVh deuten darauf hin, dass ein Mangel an körperlicher Aktivität, Übergewicht bzw. Adipositas und Rauchen das Risiko eines frühzeitigen Erwerbsausstiegs aufgrund Erwerbsunfähigkeit begünstigen können [8, 16, 20, 29]. 
Aufbauend auf diesen Erkenntnissen untersucht dieser Beitrag in einer repräsentativen Studie die Effekte der GVh körperliche Aktivität, Körpergewicht und Rauchverhalten auf die Bewertung des (noch arbeiten) Könnens älterer Beschäftigter, als Teilaspekt der EP.

\section{Methodik}

\section{Daten und Studienteilnehmer}

Analysiert wurden die Daten von 3368 älteren Beschäftigten, die 2018 an der dritten Befragungswelle der lidA(leben in der Arbeit)-Studie teilgenommen hatten und zum Erhebungszeitpunkt in einem Arbeitsverhältnis mit mindestens einer Stunde pro Woche tätig waren. lidA erforscht longitudinal die Zusammenhänge von Arbeit, Alter, Gesundheit und Erwerbsteilhabe an Beschäftigten der Geburtsjahrgänge 1959 und 1965. Die Stichprobenziehung erfolgte auf Basis der Beschäftigtenhistorik der Integrierten Erwerbsbiografien (IEB) des Instituts für Arbeitsmarktund Berufsforschung (IAB). Grundgesamtheit für die Studie sind Personen, die gemäß der Beschäftigtenhistorik am 31.12.2009 sozialversicherungspflichtig beschäftigt waren. Dies umfasst etwa $80 \%$ der gesamten Erwerbsbevölkerung hierzulande. Nicht eingeschlossen sind Beamte und Selbstständige [15, 32]. Die Stichprobe ist repräsentativ für die sozialversicherungspflichtig Beschäftigten dieser beiden Alterskohorten [32]. In regelmäßigen Abständen werden die Studienteilnehmerinnen und -teilnehmer zu Hause mittels computergestützter Interviews („computerassisted personal interviews" [CAPI]) $\mathrm{zu}$ verschiedenen Aspekten ihrer Arbeit, Gesundheit, des Privatlebens und ihrer Erwerbsperspektive befragt. Bisher fanden drei Befragungswellen statt. An der ersten Befragung 2011 nahmen 6585 Personen teil. Von den Befragten gaben 5618 (85,3\%) schriftlich ihr Einverständnis („informed consent“), sie für Folgeerhebungen wieder kontaktieren zu dürfen. Somit konnten $2014 \mathrm{im}$ Rahmen der zweiten Befragung 4244 Personen $(64,5 \%)$ und 2018 im Rahmen der dritten Befragung 3568 (54,2\%) erneut interviewt werden. Eine ausführliche Beschreibung der Konzeptualisierung und des Designs der Studie wird an anderer Stelle gegeben $[15,32]$.

\section{Variablen}

\section{Subjektive Erwerbsperspektive (EP).}

Die Befragten sollten angeben, bis $\mathrm{zu}$ welchem Alter (Altersangabe numerisch in Jahren) sie glaubten, arbeiten $\mathrm{zu}$ können. Als gültig wurden alle Altersangaben gezählt, die das tatsächliche Alter zum Befragungszeitpunkt nicht unterschritten (1959 Geborene ab 58 Jahre; 1965 Geborene ab 52 Jahre).

\section{Gesundheitsbezogene Verhaltenswei-} sen (GVh). Untersucht wurden die körperliche Aktivität in der Freizeit, das Körpergewicht und Rauchverhalten. Die körperliche Aktivität wurde anhand der Frage erfasst, an wie vielen Tagen pro Woche die Teilnehmer sich in ihrer Freizeit für mindestens 30 Minuten körperlich so anstrengten, dass sie ins Schwitzen oder außer Atem kommen. Die Formulierungsergänzung ins Schwitzen oder außer Atem kommen sollte die Befragten bei ihrer Interpretation für eine mindestens mäßig anstrengende körperliche Aktivität sensibilisieren, die mit einer $\mathrm{Zu}$ nahme der Herz- und Atemfrequenz assoziiert ist $[19,26]$. Die ursprünglich vier Antwortausprägungen wurden zu dreien zusammengefasst (aktiv $\geq 3$ Tage/Woche, 1-2 Tage/Woche, <1 Tag/Woche). Das selbstberichtete Körpergewicht wurde mittels Body-Mass-Index (BMI, kg/m²) in Anlehnung an die WHO-Klassifikation [42] in die Gruppen kein Übergewicht (BMI<25), Übergewicht (BMI 25<30) und Adipositas (BMI $\geq 30$ ) eingestuft. $\mathrm{Zu}$ Rauchern wurden jene gezählt, die zum Zeitpunkt der Befragung gelegentlich oder täglich rauchten (Antwortmöglichkeiten: Ich habe noch nie geraucht bis auf ganz seltenes Probieren, ich habe früher geraucht, ich habe in den letzten zwölf Monaten aufgehört zu rauchen, ich rauche zurzeit gelegentlich, ich rauche zurzeit täglich).

Kovariaten. Kontrolliert wurde für Geschlecht, Alter, berufliche Qualifikation, für körperliche Arbeitsbelastungen und
Arbeitsstress sowie für körperliche und mentale Gesundheit. Die berufliche Qualifikation wurde anhand des Anforderungsniveaus (Helfer-, Fachkraft-, Spezialisten- oder Expertentätigkeit) der derzeit ausgeübten Tätigkeit mittels der Klassifikation der Berufe 2010 (kldb2010; 5-Steller) erfasst. Nach dieser Einteilung wird für eine angelernte Helfertätigkeit generell keine berufliche Ausbildung vorausgesetzt, für eine fachliche Tätigkeit eine zwei- bis drei-jährige Berufsausbildung, für eine komplexe Spezialistentätigkeit eine Meisteroder Technikerausbildung und für eine hochkomplexe Expertentätigkeit eine Hochschulausbildung [1]. Zu den körperlichen Arbeitsbelastungen sollten die Teilnehmer beurteilen, wie häufig sie während ihrer Tätigkeit in ungünstigen körperlichen Haltungen arbeiten (gebückt, hockend, kniend, liegend oder über Kopf) und wie häufig sie schwere Lasten heben und/oder tragen (Frauen mehr als $10 \mathrm{~kg}$; Männer mehr als $20 \mathrm{~kg}$ ). Die Bedingungen wurden von den Befragten jeweils auf einer fünf-stufigen Skala (nie-mehr als ein Dreiviertel der Zeit) bewertet. Für die Analysen wurden die Werte beider Items gemittelt (Spanne: 0-4). Die interne Konsistenz ist akzeptabel (Cronbachs Alpha =0,69). Arbeitsstress wurde anhand der Kurzfassung des Modells zur beruflichen Gratifikationskrise (Effort Reward Imbalance [ERI]; [34]; modifizierte Version) untersucht. Die Befragten wurden nach dem Auftreten potenziell verausgabender Arbeitsbedingungen („efforts“; 3 Items zu Arbeitsunterbrechungen, Quantitative Anforderungen, Zeitdruck) und belohnender Arbeitsbedingungen („rewards“; 7 Items, u.a. Entwicklungsmöglichkeiten, Anerkennung durch den Vorgesetzten) gefragt. Bei Vorliegen eines „effort“ bzw. bei Fehlen eines „reward“ sollten die Befragten ihre daraus empfundene Belastung auf einer vier-stufigen Skala (gar nicht-sehr stark) einschätzen. Das Verhältnis von „efforts“ und „rewards“ wurde dann mittels der Summenskalen beider Dimensionen gebildet (Spanne „efforts“: 3-12; Spanne „rewards“: 7-28). Der Quotient wurde um den Faktor 7/3 gewichtet, um die Differenz der Itemanzahl von „efforts“ und „rewards“ zu 
Zbl Arbeitsmed 2021 · 71:157-166 https://doi.org/10.1007/s40664-021-00422-0

(c) Der/die Autor(en) 2021

D. Borchart · J.-B. du Prel · H. M. Hasselhorn

\section{Effekte gesundheitsrelevanter Verhaltensweisen auf die subjektive Erwerbsperspektive älterer Beschäftigter in Deutschland}

\section{Zusammenfassung}

Hintergrund und Ziel. Die subjektive Erwerbsperspektive (EP) gilt als prädiktiv für die realisierte Erwerbsdauer. Inwieweit spezifische gesundheitsrelevante Verhaltensweisen (GVh) mit der EP älterer Beschäftigter assoziiert sind, wurde bislang nicht erforscht. Vor diesem Hintergrund wurden die Effekte von körperlicher Aktivität, Übergewicht und Rauchen auf die EP älterer Beschäftigter untersucht.

Material und Methoden. Analysiert wurden Daten von 3368 älteren Erwerbstätigen, die 2018 an der dritten Befragungswelle der repräsentativen lidA-Studie teilnahmen. EP wurde anhand der Selbsteinschätzung, wie lange man glaubt, noch arbeiten zu können, untersucht. Mittels hierarchischer linearer Regressionsmodelle wurden die
Effekte der GVh auf die EP kontrolliert für soziodemografische, arbeits- und gesundheitsbezogene Faktoren querschnittlich untersucht. Durch Interaktionstestung wurde das Zusammenspiel unabhängiger Variablen auf die EP geprüft.

Ergebnisse. Körperliche Aktivität und Adipositas waren signifikant mit der EP assoziiert. Regelmäßig körperlich Aktive gehen davon aus, um fünf Monate länger arbeiten zu können als wenig bzw. nicht Aktive. Beschäftigte ohne Übergewicht gehen davon aus, um fünf Monate länger arbeiten zu können als Adipöse. Kontrolliert für die Gesundheit war der Effekt von Adipositas nicht mehr signifikant. Das Rauchverhalten zeigte keinen signifikanten direkten Effekt auf die EP.
Schlussfolgerung. Unsere Ergebnisse lassen darauf schließen, dass besonders körperliche Aktivität förderlich für die EP sein kann. Um das Potenzial der spezifischen GVh für die Erwerbsfähigkeit genauer bewerten zu können, sind weitere Untersuchungen notwendig. Betriebliche Akteure sollten die Ergebnisse bei der Planung verhaltensund verhältnispräventiver Maßnahmen berücksichtigen, um gezielter auf die Bedürfnisse der Beschäftigten, besonders jener mit potenziell kürzerer EP, eingehen zu können.

Schlüsselwörter

lidA-Studie · Ältere Arbeitnehmer · Gesundheitsverhalten · Erwerbsfähigkeit . Verhaltensprävention

\section{Effects of health behaviors on the subjective employment perspective of older workers in Germany}

\section{Abstract}

Background and aim. The subjective employment perspective (EP) is considered an important predictor for the actual duration of labor market participation. So far, no research has focused on the association of specific health behaviors (HB) and the EP of older workers. The effects of physical activity, being overweight and smoking on EP of older workers were therefore examined.

Material and methods. Data from 3368 older employees, who participated in the third survey wave of the representative lidA study in 2018 were analyzed. EP was measured by the employees' estimation on how long they think they can continue working. By means of hierarchical linear regression, the effects of HB-factors on EP were cross-sectionally examined, controlling for sociodemographic, work-related and health-related factors. Interaction terms were tested in order to investigate the interplay of independent variables on EP.

Results. Physical activity and obesity were significantly associated with EP. The EP of employees with regular physical activity is five months longer than less active or nonactive employees. The EP of normal weight employees is five months longer than obese employees. Regarding health aspects, the effect of obesity on EP lost significance. Smoking did not show any significant direct effect on EP.
Conclusion. The results indicate that regular physical activity in particular can be beneficial for EP. Further research is needed to improve the potential of specific HB for EP in depth. These findings should be considered by occupational stakeholders in behavioral and contextual preventive measures in order to respond more specifically to employees' needs, especially those with a potentially shorter EP.

\section{Keywords}

lidA study - Older workers - Health habits . Fitness to work - Behavior-based prevention berücksichtigen (gewichtetes Verhältnis: ERI-R). Die körperliche Gesundheit und die mentale Gesundheit wurden anhand der Short Form-12 Health Survey (SF-12)-Subskalen Physical health und Mental health (SF12-v2 Sozio-oekonomischer Panel (SOEP) Version; [24]) untersucht. Niedrigere Werte bedeuten einen schlechteren Gesundheitszustand.

\section{Fehlende Werte}

Von den 3368 älteren Beschäftigten, die zum Zeitpunkt der Befragung (2018) erwerbstätig und nicht verrentet waren, lagen 3137 gültige Angaben zur EP vor (93,1\%). Die Anzahl fehlender Werte zu den GVh-Faktoren und Kovariaten reichte von 0 bis 367. Die fehlenden Werte wurden mittels multipler Imputation mit der Fully-ConditionalSpecification(FCS)-Methode [4] ersetzt. Hierdurch erhöhte sich die Anzahl ein- geschlossener Fälle im gemeinsamen multiplen Analysemodell von 2718 auf 3365.

\section{Analysen}

Mit multiplen linearen Regressionsmodellen wurden die Effekte der GVh und Kovariaten auf die EP unter blockweiser Hinzunahme der Variablen in das Modell (Modelle 0-3) untersucht. So wurden zuerst die Effekte der GVh-Faktoren auf die EP ohne Kontrolle der Ko- 
Tab. 1 Deskriptive Charakteristika der Studienteilnehmer $(N=3368)$

\begin{tabular}{|c|c|c|}
\hline & $N$ & $M(S D)$ \\
\hline Erwerbsperspektive (Alter in Jahren) ${ }^{\mathrm{a}}$ & 3137 & $65,15(3,77)$ \\
\hline Körperliche Arbeitsexposition (Spanne 0-4) & 3339 & $0,65(0,84)$ \\
\hline Arbeitsstress (ERI-R; Spanne 0,2-5,0) ${ }^{c}$ & 3001 & $0,65(0,40)$ \\
\hline Körperliche Gesundheit (Spanne 0-100) ${ }^{d}$ & 3332 & $48,01(9,20)$ \\
\hline \multirow[t]{2}{*}{ Mentale Gesundheit (Spanne 0-100) ${ }^{d}$} & 3332 & $51,67(9,89)$ \\
\hline & & $n(\%)$ \\
\hline Männer ${ }^{\mathrm{e}}$ & 3368 & $1527(45,3)$ \\
\hline 1959 Geborene $^{f}$ & 3368 & $1499(44,5)$ \\
\hline Berufliches Anforderungsniveau & 3315 & \\
\hline Helfer (an-/ungelernte Tätigkeit) & & $241(7,3)$ \\
\hline Fachkraft (fachliche Tätigkeit) & & $1822(55,0)$ \\
\hline Spezialist (komplexe Tätigkeit) & & $627(18,9)$ \\
\hline Experte (hoch komplexe Tätigkeit) & & $625(18,9)$ \\
\hline Körperliche Aktivität & 3366 & \\
\hline 3 oder mehr Tage/Woche & & $1153(34,3)$ \\
\hline 1-2 Tage/Woche & & $1494(44,4)$ \\
\hline$<1$ Tag/Woche & & $719(21,4)$ \\
\hline Body-Mass-Index (BMI) & 3284 & \\
\hline Kein Übergewicht (BMI<25) & & $1007(30,7)$ \\
\hline Übergewicht (BMI $25<30$ ) & & $1425(43,4)$ \\
\hline Adipositas (BMI $\geq 30)$ & & $852(25,9)$ \\
\hline Raucherg & 3366 & $923(27,4)$ \\
\hline \multicolumn{3}{|c|}{ 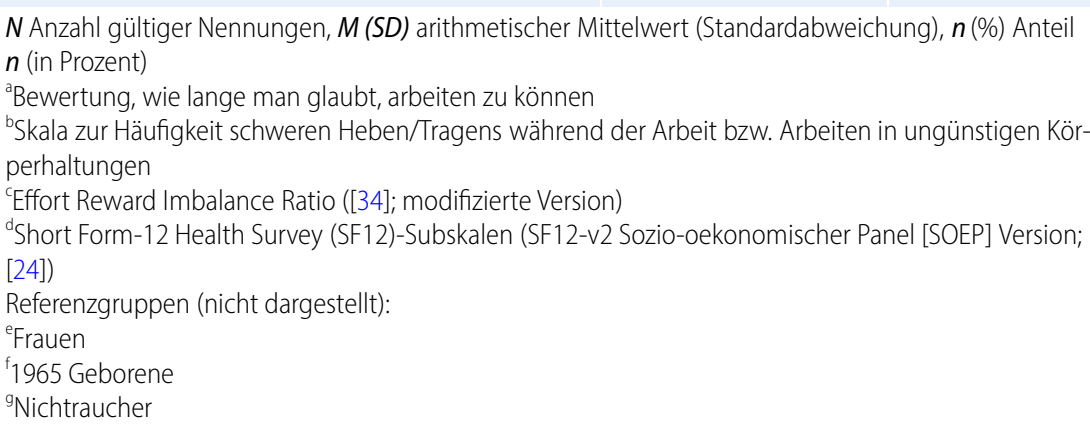 } \\
\hline
\end{tabular}

variaten geprüft (Modell 0). Dann wurden die GVh-Effekte auf die EP unter Hinzunahme der soziodemografischen Aspekte geprüft (Modell 1). Im nächsten Schritt wurden die Arbeitsbedingungen als weitere Kovariaten in das Modell mitaufgenommen (Modell 2). Abschließend erfolgte die Hinzunahme der Gesundheitsvariablen in das Modell (Modell 3). Durch dieses Vorgehen wurde ersichtlich, inwieweit sich die Regressionskoeffizienten der GVh unter Kontrolle der aufgenommenen Variablen verändern und inwieweit die aufgenommenen Variablen zur Varianzaufklärung der EP beitragen. Das gemeinsame Modell 3 wurde zudem auf Interaktionen der GVh mit der körperlichen Gesundheit und mentalen Ge-

\section{Ergebnisse}

\section{Stichprobenbeschreibung}

Die Mehrheit der Befragten war weiblich, 1965 geboren und beruflich als Fachkraft tätig. Im Mittel gingen die Befragten davon aus, dass sie bis zu einem Alter von 65 Jahren und zwei Monaten $(M=65,15$, $\mathrm{SD}=3,77)$ arbeiten können. Die Mehrheit von ihnen war zu wenig körperlich aktiv (weniger als $1 \mathrm{Tag} /$ Woche bzw. 1-2 Tage/Woche $=65,7 \%)$, übergewichtig bzw. adipös $(69,3 \%)$ und gut ein Viertel rauchte. $\bullet$ Tab. 1 stellt die deskriptiven Charakteristika der Stichprobe dar.

\section{Analysemodelle}

Bis auf das Rauchverhalten waren alle getesteten GVh und Kovariaten in den bivariaten Modellen (nicht dargestellt) signifikant mit der EP assoziiert. Multikollinearität der Prädiktoren konnte anhand der Korrelationsanalysen untereinander sowie der Prüfung der Toleranz und des Varianzinflationsfaktors (VIF) in den multiplen Analysemodellen ausgeschlossen werden. - Tab. 2 stellt die linearen Regressionsmodelle dar.

\section{Zusammenhang der GVh mit der EP}

Die körperliche Aktivität war in allen Modellen signifikant mit der EP assoziiert. Kontrolliert für alle GVh und Kovariaten (Modell 3) zeigte sich, dass körperlich Aktive (mindestens 3 Tage/ Woche) davon ausgehen, vier Monate länger arbeiten zu können als mäßig Aktive $(\mathrm{B}=-0,342$ [95\% KI: -0,680--0,005]) und fünf Monate länger als wenig bzw. nicht Aktive $(\mathrm{B}=-0,378$ [95\% KI: $-0,650--0,105])$. Für das Körpergewicht zeigte sich kontrolliert für die soziodemografischen Faktoren und die Arbeitsbedingungen (Modell 1 und Modell 2), dass Adipositas signifikant mit der EP assoziiert war. Beschäftigte ohne Übergewicht $(\mathrm{BMI}<25)$ gehen davon aus, fünf Monate länger arbeiten zu können als Adipöse (Modell 2: $\mathrm{B}=-0,456$ [95\% KI: $-0,797--0,114]$ ). Der Effekt war unter Kontrolle der Gesundheitsfaktoren (Modell 3) nicht mehr 
Tab. 2 Multiple hierarchische lineare Regressionsmodelle zur Einschätzung der Befragten, bis zu welchem Alter (in Jahren) sie arbeiten können $(N=3368)$

\begin{tabular}{|c|c|c|c|c|c|c|c|c|c|c|c|c|}
\hline & \multicolumn{3}{|c|}{$\begin{array}{l}\text { Modell 0: } \\
\text { Gesundheitsrelevante } \\
\text { Verhaltensweisen }\end{array}$} & \multicolumn{3}{|c|}{$\begin{array}{l}\text { Modell 1: } \\
\text { Modell } 0 \text { und sozio- } \\
\text { demografische Faktoren }\end{array}$} & \multicolumn{3}{|c|}{$\begin{array}{l}\text { Modell 2: } \\
\text { Modell } 1 \text { und Arbeitsbe- } \\
\text { dingungen }\end{array}$} & \multicolumn{3}{|c|}{$\begin{array}{l}\text { Modell 3: } \\
\text { Modell } 2 \text { und Gesund- } \\
\text { heitsvariablen }\end{array}$} \\
\hline & B & $\mathbf{K I}-$ & $\mathbf{K I}+$ & B & KI - & $\mathbf{K I}+$ & B & $\mathbf{K I}$ - & $\mathbf{K I}+$ & B & $\mathbf{K I}$ - & $\mathbf{K I}+$ \\
\hline \multicolumn{13}{|l|}{ Körperliche Aktivität ${ }^{\mathrm{a}}$} \\
\hline An 1-2 Tage/Woche aktiv & $-0,376^{*}$ & $-0,674$ & $-0,078$ & $-0,420^{*}$ & $-0,709$ & $-0,132$ & $-0,493^{*}$ & $-0,773$ & $-0,213$ & $-0,342^{*}$ & $-0,680$ & $-0,005$ \\
\hline An $<1$ Tag/Woche aktiv & $-0,638^{*}$ & $-1,004$ & $-0,271$ & $-0,586^{*}$ & $-0,944$ & $-0,229$ & $-0,586^{*}$ & $-0,931$ & $-0,241$ & & $-0,650$ & $-0,105$ \\
\hline \multicolumn{13}{|l|}{ Body-Mass-Index (BMI) $)^{b}$} \\
\hline Übergewicht (BMI 25 <30) & $-0,004$ & $-0,318$ & 0,311 & $-0,092$ & $-0,405$ & 0,221 & $-0,062$ & $-0,369$ & 0,245 & $-0,003$ & $-0,301$ & 0,296 \\
\hline Adipositas (BMI $\geq 30$ ) & $-0,574^{*}$ & $-0,928$ & $-0,220$ & $-0,449^{*}$ & $-0,798$ & $-0,099$ & $-0,456^{*}$ & $-0,797$ & $-0,114$ & $-0,095$ & $-0,435$ & 0,245 \\
\hline Raucher ${ }^{c}$ & $-0,162$ & $-0,459$ & 0,135 & 0,035 & $-0,255$ & 0,324 & 0,145 & $-0,135$ & 0,425 & 0,266 & $-0,007$ & 0,540 \\
\hline Männer ${ }^{d}$ & & & & $0,591^{*}$ & 0,322 & 0,861 & $0,542^{*}$ & 0,279 & 0,806 & $382^{*}$ & 0,127 & 0,638 \\
\hline 1959 Geborene $^{e}$ & & & & $0,476^{*}$ & 0,221 & 0,732 & $0,377^{*}$ & 0,127 & 0,627 & & 0,278 & 0,765 \\
\hline \multicolumn{13}{|l|}{ Berufliches Anforderungsniveau ${ }^{f}$} \\
\hline Helfer (an-/ungelernte Tätigkeit) & & & & $-0,485$ & $-0,986$ & 0,017 & $-0,558^{*}$ & $-1,054$ & $-0,062$ & $-0,277$ & $-0,758$ & 0,204 \\
\hline Spezialist (komplexe Tätigkeit) & & & & $1,117^{*}$ & 0,779 & 1,455 & $0,813^{*}$ & 0,479 & 1,148 & $0,633^{*}$ & 0,308 & 0,957 \\
\hline Experte (hochkomplexe Tätigkeit) & & & & $2,016^{*}$ & 1,657 & 2,375 & $1,587^{*}$ & 1,226 & 1,948 & $1,363^{*}$ & 1,008 & 1,718 \\
\hline Körperliche Arbeitsexposition ${ }^{g}$ & & & & & & & $-0,688^{*}$ & $-0,849$ & $-0,527$ & $-0,498^{*}$ & $-0,658$ & $-0,339$ \\
\hline Arbeitsstress (ERI-R) ${ }^{\mathrm{h}}$ & & & & & & & $-1,810^{*}$ & $-2,140$ & $-1,479$ & $-1,095^{*}$ & $-1,435$ & $-0,754$ \\
\hline Körperliche Gesundheit ${ }^{\mathrm{i}}$ & & & & & & & & & & $0,091^{*}$ & 0,077 & 0,106 \\
\hline Mentale Gesundheit ${ }^{i}$ & & & & & & & & & & $0,058^{*}$ & 0,045 & 0,071 \\
\hline Modellzusammenfassung & \multicolumn{3}{|c|}{$\begin{array}{l}\text { Korr. } R^{2}=0,010 \\
F(5,3360)=6,638^{*}\end{array}$} & \multicolumn{3}{|c|}{$\begin{array}{l}\text { Korr. } R^{2}=0,074 \\
F(10,3355)=26,802^{*}\end{array}$} & \multicolumn{3}{|c|}{$\begin{array}{l}\text { Korr. } R^{2}=0,138 \\
F(12,3353)=44,642^{*}\end{array}$} & \multicolumn{3}{|c|}{$\begin{array}{l}\text { Korr. } R^{2}=0,189 \\
F(14,3351)=56,842^{*}\end{array}$} \\
\hline \multicolumn{13}{|c|}{ 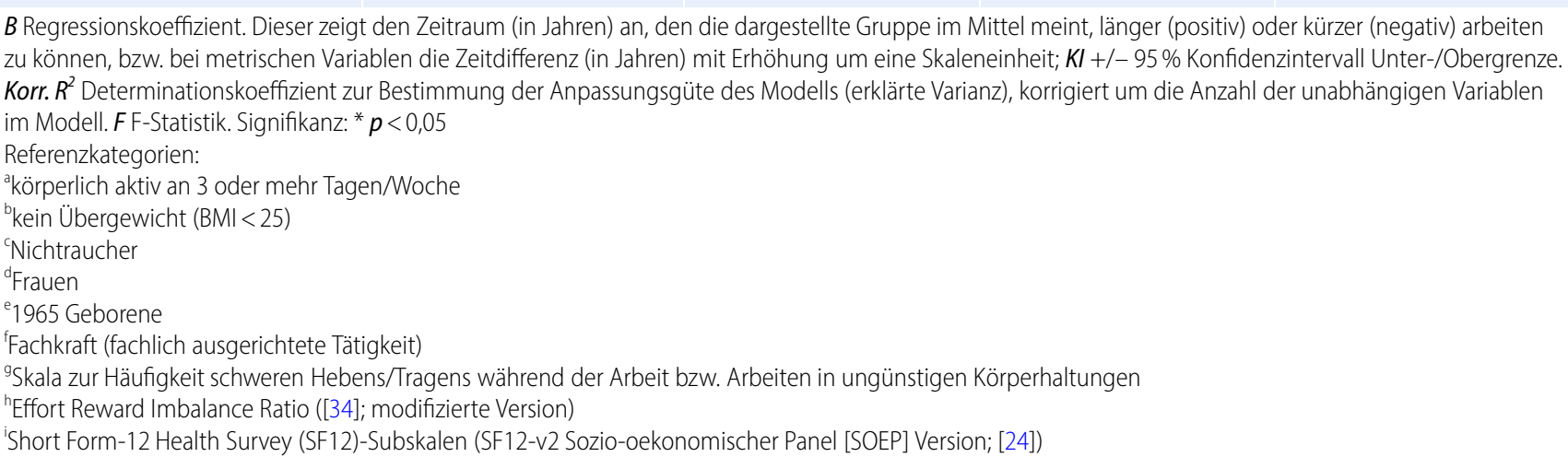 } \\
\hline
\end{tabular}

signifikant. Das Rauchverhalten zeigte keinen signifikanten direkten Effekt auf die EP.

Modell 0 war signifikant, allerdings konnten hierbei die GVh nur einen geringen Teil der Varianz erklären (korr. $\left.\mathrm{R}^{2}=0,010 ; \mathrm{F}(5 ; 3365)=6,638 ; p<0,05\right)$. Unter Kontrolle der soziodemografischen Faktoren (Vergleich Modell $0 \mathrm{zu}$ Modell 1), verringerte sich der Effekt für geringe körperliche Aktivität (weniger $1 \mathrm{Tag} /$ Woche) und von Adipositas. Der Effekt für mäßig Aktive verstärkte sich kontrolliert für die soziodemografischen Faktoren und Arbeitsbedingungen (Modell $0 \mathrm{zu}$ Modell $1 \mathrm{zu}$ Modell 2). Kontrolliert für die Gesundheitsvaria- blen verringerten sich die Effekte beider körperlichen Aktivitätsgruppen (Modell $2 \mathrm{zu}$ Modell 3). In den durchgeführten Interaktionstestungen zeigte sich ein signifikanter Effekt für die Interaktion Rauchen mit körperlicher Gesundheit $(\mathrm{B}=0,047$ [95\% KI: 0,016 - 0,077]). Der positive Zusammenhang der körperlichen Gesundheit mit der EP ist demzufolge bei Rauchern stärker als bei Nichtrauchern. - Abb. 1 veranschaulicht grafisch den Zusammenhang der körperlichen Gesundheit mit der EP je nach Rauchverhalten (Raucher vs. Nichtraucher).

Unter Einschluss dieser Interaktion in das Modell wurde auch der direkte Effekt des Rauchverhaltens auf die EP signifikant (B= 0,296 [95\% KI: 0,034-0,569]). - Tab. 3 stellt das signifikante Interaktionsmodell dar.

Zur weiteren Untersuchung der signifikanten Interaktion wurden Simple Slope Testungen ([2]; nicht dargestellt) durchgeführt. In diesen zeigte sich, dass Rauchen lediglich bei guter körperlicher Gesundheit (+ 1 SD) die EP direkt beeinflusst ( $B=0,725$ [95\% KI: 0,328 - 1,122). Bei guter körperlicher Gesundheit gehen Raucher davon aus, etwa neun Monate länger als die Nichtraucher arbeiten $\mathrm{zu}$ können. 


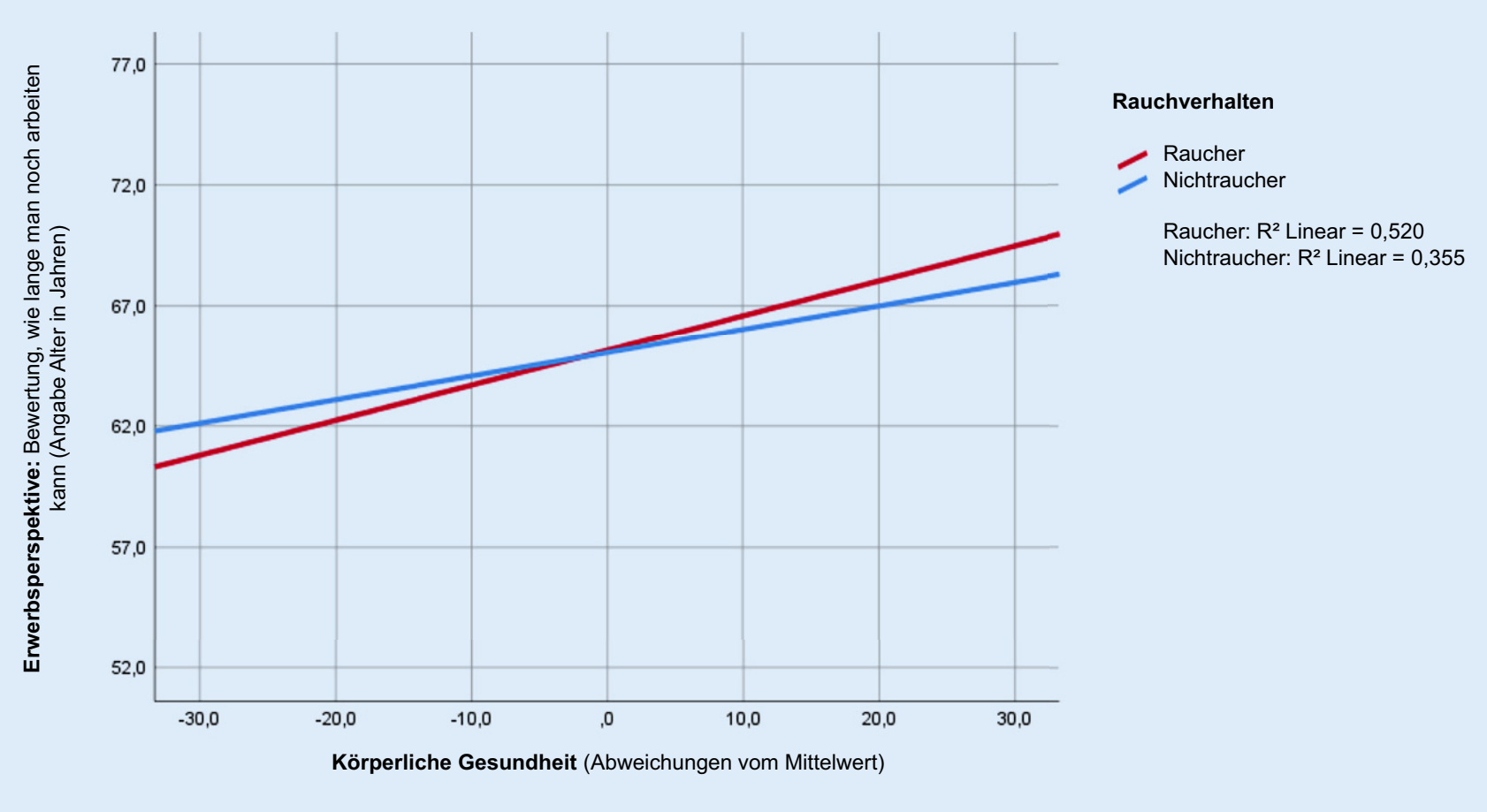

Abb. 1 \& Interaktion Rauchen × körperliche Gesundheit

\section{Zusammenhang der Kovariaten mit der EP}

Alle Kovariaten waren über die Modelle hinweg signifikant mit der EP assoziiert. Im gemeinsamen Modell 3 zeigte sich, dass Männer und Ältere (1959 Geborene) im Vergleich zu den Übrigen davon ausgehen, fünf bzw. sechs Monate länger arbeiten zu können (Männer: $\mathrm{B}=0,382$ [95\% KI: 0,127 - 0,638]; 1959 Geborene: $\mathrm{B}=0,521$ [95\% KI: 0,278-0,765]). Spezialisten berichteten eine um acht Monate ( $\mathrm{B}=0,633$ [95\% KI: 0,308 - 0,957]), Experten eine um 16 Monate $(B=1,363$ [95\% KI: 1,008 - 1,718]) längere EP als Fachkräfte. Der Unterschied in der EP von Fachkräften zu Beschäftigten mit Helfertätigkeiten war im gemeinsamen Modell 3 nicht signifikant. Körperliche Arbeitsbelastungen $(B=-0,498 \quad[95 \%$ KI: $-0,658--0,339])$ und Arbeitsstress $(\mathrm{B}=-1,095$ [95\% KI: $-1,435--0,754])$ waren erwartungskonform negativ mit der EP assoziiert. Körperliche Gesundheit $(B=0,091$ [95\% KI: $0,077-0,106])$ und mentale Gesundheit ( $\mathrm{B}=0,058$ [95\% KI: $0,045-0,071])$ zeigten erwartungskonform einen positiven Effekt auf die EP.

\section{Diskussion}

Die Ergebnisse dieser Untersuchung lassen darauf schließen, dass spezifische GVh (hier: körperliche Aktivität, Körpergewicht, Rauchverhalten) für die EP älterer Beschäftigter von Bedeutung sein können - auch wenn sie diese nur bedingt erklären. Im Schnitt gingen die Befragten davon aus, bis $\mathrm{zu}$ einem Alter von 65 Jahren und zwei Monaten arbeiten zu können. Vergleichbar mit den Ergebnissen anderer repräsentativer Studien zu GVh von Personen im mittleren Erwachsenenalter [9, 19, 31, 44] zeigte sich, dass mehr als die Hälfte der befragten Alterskohorten zu wenig körperlich aktiv, übergewichtig bzw. adipös ist und etwa ein Viertel raucht.

Übereinstimmend mit den Ergebnissen früherer Studien zum Zusammenhang von GVh mit einem gesundheitsbedingten Erwerbsausstieg [8, 16, 20, 29] waren die körperliche Aktivität und Adipositas signifikant mit der EP assoziiert. Unter Berücksichtigung aller getesteten soziodemografischen, arbeitsbezogenen und gesundheitlichen Faktoren haben wenig bzw. nicht Aktive eine um etwa fünf Monate kürzere EP als aktive Personen. Ferner zeigte sich, dass Adipöse eine um fünf Monate kürzere EP berichteten als Personen ohne Übergewicht. Dieser Effekt war allerdings kontrolliert für die körperliche und mentale Gesundheit nicht mehr signifikant.

Unter Einschluss der soziodemografischen Variablen (Modell 0 zu Modell 1) verringerte sich der Effekt für die Subgruppen wenig bzw. nicht Aktive und Adipositas auf die EP. So ist davon auszugehen, dass diese soziodemografischen Variablen einen Teil des Unterschiedes im arbeiten Können bei wenig bzw. nicht Aktiven und bei Adipösen erklären. Der Effekt für die Gruppe mäßig Aktiver verstärkte sich über die Modelle hinweg. Demnach modifizieren die untersuchten Kovariaten in dieser Aktivitätsgruppe offenbar den Einfluss auf die EP. Dies deutet wiederum auf ein komplexes Zusammenspiel der Kovariaten mit der körperlichen Aktivität im Einfluss auf die EP. So können Unterschiede in der Art und Weise der körperlichen Aktivität zwischen den untersuchten Personengruppen möglicherweise die unterschiedliche Bewertung der EP erklären. Es ist z. B. bekannt, dass sich Frauen und Männer in ihrer Wahl der Sportarten und der Intensitätsausübung unterscheiden [27]. Dem Erklärungsgehalt dieser Annahme kann 
Tab. 3 Gemeinsames Analysemodell (Modell 3) inklusive der Interaktion Rauchen und körperliche Gesundheit $(N=3368)$

\begin{tabular}{|c|c|c|c|}
\hline & \multicolumn{3}{|c|}{ Interaktionsmodell } \\
\hline & B & $\mathbf{K I}-$ & $\mathbf{K I}+$ \\
\hline \multicolumn{4}{|l|}{ Körperliche Aktivität ${ }^{a}$} \\
\hline An 1-2 Tage/Woche aktiv & $-0,363^{*}$ & $-0,700$ & $-0,025$ \\
\hline An $<1$ Tag/Woche aktiv & $-0,384^{*}$ & $-0,656$ & $-0,112$ \\
\hline \multicolumn{4}{|l|}{ Body-Mass-Index (BMI) ${ }^{b}$} \\
\hline Übergewicht (BMI $25<30$ ) & $-0,013$ & $-0,311$ & 0,285 \\
\hline Adipositas (BMI $\geq 30)$ & $-0,119$ & $-0,459$ & 0,222 \\
\hline Raucher ${ }^{c}$ & $0,296^{*}$ & 0,023 & 0,569 \\
\hline Männer ${ }^{d}$ & $0,395^{*}$ & 0,140 & 0,650 \\
\hline 1959 Geborene $^{\mathrm{e}}$ & $0,519^{*}$ & 0,276 & 0,762 \\
\hline \multicolumn{4}{|l|}{ Berufliches Anforderungsniveau ${ }^{f}$} \\
\hline Helfer (an-/ungelernte Tätigkeit) & $-0,288$ & $-0,769$ & 0,192 \\
\hline Spezialist (komplexe Tätigkeit) & $0,640^{*}$ & 0,316 & 0,964 \\
\hline Experte (hochkomplexe Tätigkeit) & $1,369^{*}$ & 1,014 & 1,724 \\
\hline Körperliche Arbeitsexposition ${ }^{g}$ & $-0,487^{*}$ & $-0,646$ & $-0,327$ \\
\hline Arbeitsstress (ERI-R) ${ }^{\mathrm{h}}$ & $-1,094^{*}$ & $-1,434$ & $-0,754$ \\
\hline Körperliche Gesundheit ${ }^{i}$ & $0,078^{*}$ & 0,062 & 0,095 \\
\hline Mentale Gesundheit ${ }^{i}$ & $0,058^{*}$ & 0,045 & 0,071 \\
\hline Interaktion Rauchen × körperliche Gesundheit & $0,047^{*}$ & 0,016 & 0,077 \\
\hline Modellzusammenfassung & \multicolumn{3}{|c|}{$\begin{array}{l}\text { Korr. } R^{2}=0,194 \\
F(15,3350)=53,904^{*}\end{array}$} \\
\hline \multicolumn{4}{|c|}{ 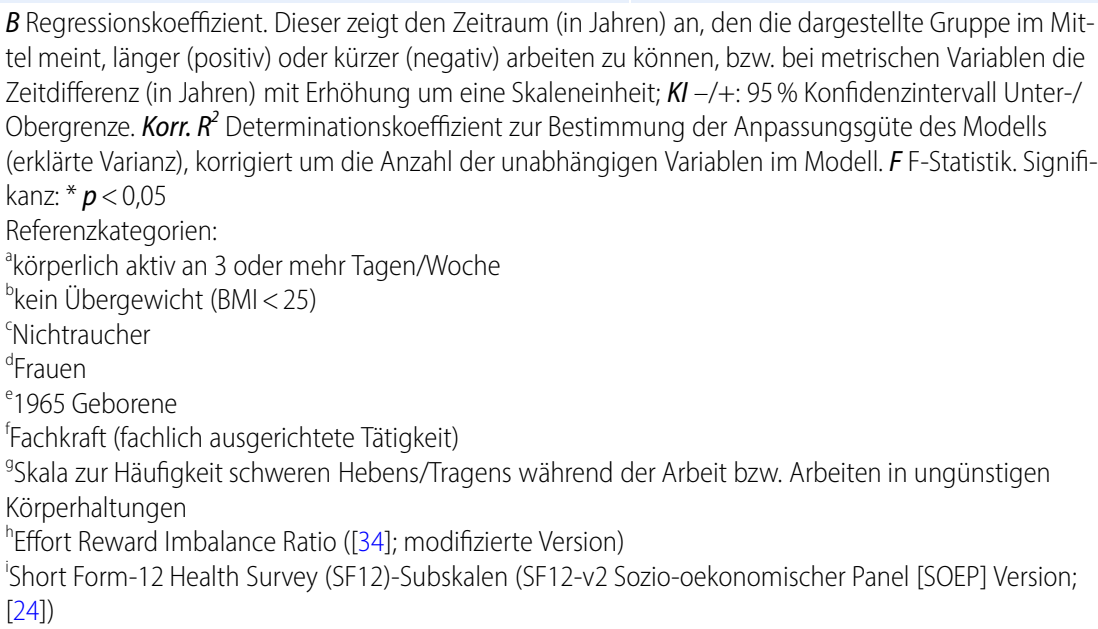 } \\
\hline
\end{tabular}

aufgrund der herangezogenen Parametrisierung der körperlichen Aktivität an dieser Stelle nicht nachgegangen werden.

Der signifikante Effekt von Adipositas war unter Kontrolle der Gesundheitsvariablen nicht mehr signifikant. Adipöse haben in unserer Studie eine deutlich kürzere EP als Personen ohne Übergewicht, was aber statistisch nahezu vollständig mit ihrer schlechteren Gesundheit erklärt werden kann.

Das Rauchverhalten war in den Analysemodellen nicht mit der EP assoziiert. Unter Einschluss der signifikanten In- zen häufig das relative Gesundheitsrisiko des Tabakkonsums [18]. Denkbar ist daher, dass Raucher wegen des vorliegenden Risikofaktors ihre EP stärker mit Blick auf ihre körperliche Gesundheit bewerten, die EP bei wahrgenommener guter körperlicher Gesundheit aber eher überschätzen. Dies sollten weitere Studien prüfen.

Bezüglich der festgestellten Zusammenhänge der Kovariaten mit der EP stehen die Befunde in Übereinstimmung zu Berichten in Übersichtsarbeiten, nach denen Männer und Beschäftigte in höheren beruflichen Tätigkeiten länger erwerbstätig sind [30, 41]. Dies gilt ebenso für unsere Befunde, nach denen körperliche Arbeitsbelastungen und Arbeitsstress mit einer kürzeren EP einhergehen und körperliche und mentale Gesundheit mit einer längeren. Der signifikante Altersunterschied bei der EP ist konsistent mit Ergebnissen anderer Untersuchungen zur selbstberichteten EP zu sehen [13, 22, 23, $25,43]$. Es ist anzunehmen, dass mit zunehmendem Alter sich der Einfluss der gegenwärtigen Lebens- und Arbeitsunterschiede auf die EP wandelt [13, 22]. Auch verringert sich der Prognosezeitraum mit zunehmendem Alter, was die EP beeinflussen kann [13]. Allerdings kann es auch sein, dass der Altersunterschied auf die Zugehörigkeit zur Geburtskohorte zurückzuführen ist. Hierbei können beispielsweise verschiedene Normen (z.B. unterschiedliche Betroffenheit durch Bestimmungen zur gesetzlichen Regelaltersgrenze des Renteneintritts) und Werte (z. B. Stellung der Arbeit im persönlichem Leben) bei der Bewertung der EP von Bedeutung sein [13]. Aufgrund des querschnittlichen Untersuchungsdesigns und der Befragung lediglich älterer Beschäftigter ist nicht eindeutig zu beurteilen, ob der signifikante Altersunterschied ein Alters- oder Kohorteneffekt ist.

Eine Stärke dieser Untersuchung ist die Repräsentativität der Studienpopulation für die sozialversicherungspflichtig Beschäftigten der Geburtsjahrgänge 1959 und 1965 hier in Deutschland [32]. Sie ermöglicht es, Rückschlüsse auf die Verteilungen und Zusammenhänge der Faktoren für Beschäftigte dieser beiden Alterskohorten zu ziehen. Die Generalisierbar- 
keit der Ergebnisse auf die Gesamtheit aller älteren sozialversicherten Beschäftigten ist aber durch die Befragung von nur zwei Geburtsjahrgängen eingeschränkt. Eine weitere Stärke der Untersuchung stellt die Größe der Stichprobe dar. Sie ermöglicht die multiple Testung von Unterschieden in Subgruppen (z. B. Raucher mit guter körperlichen Gesundheit). Die multiple Imputation mittels FCS-Methode sehen wir als weitere Stärke an. Dadurch erhöhte sich die Anzahl der im multiplen Analysemodell eingeschlossenen Fälle von 2718 auf 3365. Berechnete Analysemodelle ohne Imputation zeigten sehr ähnliche Ergebnisse. Wegen des querschnittlichen Untersuchungsdesigns sind kausale Wirkzusammenhänge vorsichtig zu betrachten. Die untersuchten GVh wurden subjektiv erfragt. Daher ist nicht auszuschließen, dass die Angaben einer Verzerrung aufgrund sozialer Erwünschtheit unterliegen, bzw. einer Abweichung zum tatsächlichen GVh aus anderen Gründen. Nichtsdestotrotz sind die Verteilungshäufigkeiten der körperlichen Aktivität, des Körpergewichts und des Rauchverhaltens der befragten Alterskohorten vergleichbar mit denen anderer repräsentativer Studien zu GVh von Personen im mittleren Erwachsenenalter $[9,19,31,44]$.

\section{Schlussfolgerung und Ausblick}

Die Alterung und Schrumpfung der Bevölkerung machen es aus sozialpolitischer wie wirtschaftlicher Sicht möglicherweise unerlässlich, dass ältere $\mathrm{Be}$ schäftigte länger arbeiten müssen. Die Befragten dieser repräsentativen Studie gingen im Schnitt aber davon aus, bis zu einem Alter von 65 Jahren und zwei Monaten arbeiten zu können. Dies unterschreitet das derzeitige Regelrenteneintrittsalter von 66 bzw. 67 Jahren für die untersuchten Alterskohorten. Wenn nun die Bewertung, wie lange man noch arbeiten kann, relevanter Prädiktor für den tatsächlichen Erwerbsausstieg ist, sollten beeinflussende Faktoren dieser Einschätzung für die rechtzeitige Planung von Gegenmaßnahmen von Interesse sein.

In diesem Beitrag ging es darum, $\mathrm{zu}$ untersuchen, inwieweit spezifische GVh mit der Bewertung des eigenen (noch arbeiten) Könnens älterer Beschäftigter zusammenhängen. Die hier festgestellten Zusammenhänge weisen darauf hin, dass für diesen Teilaspekt der EP die untersuchten GVh bis zu einem gewissen Grad von Bedeutung sein können.

Die Effekte der GVh auf die Bewertung des eigenen (noch arbeiten) Könnens sind als gering einzustufen. Dabei ist allerdings zu bedenken, dass auch die anderen untersuchten Merkmale wie Geschlecht, körperliche Arbeitsbelastungen und Gesundheit die EP nur eingeschränkt erklärten. So deuten die Analysen auf ein komplexes Zusammenwirken derverschiedenen individuellen und kontextualen Faktoren bei der Bewertung des (noch arbeiten) Könnens hin. Nichtsdestotrotz zeigte sich, dass die körperliche Aktivität unabhängig vom Gesundheitszustand und allen anderen untersuchten Faktoren, die EP signifikant begünstigen kann.

Zukünftige Studien sollten die Zusammenhänge der EP mit den hier untersuchten GVh und Kovariaten längsschnittlich überprüfen. Die Überprüfung der Effekte von Veränderungen der GVh über die Zeit auf die EP kann für Interventionen interessant sein. Die Gründe, warum körperliche Aktivität kontrolliert für alle anderen untersuchten Variablen, einen signifikanten positiven Effekt auf die EP hat, sollte in qualitativen Untersuchungen näher untersucht werden. Dies kann einen tieferen Einblick in das mögliche Potenzial dieser GVh für die Erwerbsteilhabe geben. Es ist durchaus denkbar, dass die Förderung von körperlicher Aktivität über den gesundheitlichen Nutzen hinaus zur Erwerbsteilhabe älterer Beschäftigter beitragen kann. Unternehmen sollten die gefundenen Ergebnisse daher nutzen, um gezielter auf individuelle Pläne und Bedarfe, besonders von Beschäftigten mit potenziell kürzeren EP, eingehen zu können. So können betriebliche Maßnahmen zur Gesundheitsförderung und Prävention, die individuelle und kontextuale Prädiktoren der subjektiven EP gezielt berücksichtigen, möglicherweise über den gesundheitlichen Nutzen hinaus dazu beitragen, dass Beschäftigte meinen, länger arbeiten zu können und es dann vielleicht auch eher tun.

\section{Fazit}

- Für die Arbeitswelt wird es zukünftig sehr bedeutend sein, Beschäftigte so lange wie möglich im Erwerb zu halten. Die subjektive Erwerbsperspektive (EP) gilt als prädiktiv für die Erwerbsdauer und sollte daher wissenschaftlich und gesellschaftlich mehr Beachtung finden.

- Als zentrale Komponente der EP wird die eigene Bewertung, wie lange man noch arbeiten kann, angesehen. Dieser Bewertung scheint ein komplexes Zusammenspiel individueller und kontextualer Faktoren zugrunde zu liegen. Um die Wirkmechanismen genauer $\mathrm{zu}$ verstehen, sollten die hier gefundenen Zusammenhänge in längsschnittlichen Untersuchungen weiter erforscht werden.

- Eine regelmäßige körperliche Aktivität (mind. 3 Tage/Woche) begünstigt die EP älterer Beschäftigter. Die Gründe hierfür sollten im Rahmen qualitativer Forschungen näher untersucht werden, um das Potenzial dieser gesundheitsrelevanten Verhaltensweise für die Erwerbsteilhabe genauer bewerten zu können.

- Unternehmen sollten die gefundenen Ergebnisse nutzen, um bei der Planung und Implementierung von Maßnahmen gezielter auf die Bedürfnisse und Belange älterer Beschäftigter eingehen zu können.

\section{Korrespondenzadresse}

Daniela Borchart, M.Sc.

Fachgebiet Arbeitswissenschaft, Bergische Universität Wuppertal

Gaußstraße 20, 42119 Wuppertal, Deutschland borchart@uni-wuppertal.de

Förderung. Die vorliegende Arbeit wurde gefördert durch die BARMER Krankenkasse im Rahmen der Förderung des lidA III-BGF Teilprojekts, Förderkennzeichen: 2016-379.

Funding. Open Access funding enabled and organized by Projekt DEAL. 


\section{Einhaltung ethischer Richtlinien}

Interessenkonflikt. D. Borchart, J.-B. du Prel und H.M. Hasselhorn geben an, dass kein Interessenkonflikt besteht.

Für diese Studie liegt das Votum der Ethikkommission der Universität Wuppertal vor (5. Dezember 2008). Von allen befragten Studienteilnehmern liegt eine informierte Einverständniserklärung (,informed consent ${ }^{\prime \prime}$ ) vor.

Open Access. Dieser Artikel wird unter der Creative Commons Namensnennung 4.0 International Lizenz veröffentlicht, welche die Nutzung, Vervielfältigung, Bearbeitung, Verbreitung und Wiedergabe in jeglichem Medium und Format erlaubt, sofern Sie den/die ursprünglichen Autor(en) und die Quelle ordnungsgemäß nennen, einen Link zur Creative Commons Lizenz beifügen und angeben, ob Änderungen vorgenommen wurden.

Die in diesem Artikel enthaltenen Bilder und sonstiges Drittmaterial unterliegen ebenfalls der genannten Creative Commons Lizenz, sofern sich aus der Abbildungslegende nichts anderes ergibt. Sofern das betreffende Material nicht unter der genannten Creative Commons Lizenz steht und die betreffende Handlung nicht nach gesetzlichen Vorschriften erlaubt ist, ist für die oben aufgeführten Weiterverwendungen des Materials die Einwilligung des jeweiligen Rechteinhabers einzuholen.

Weitere Details zur Lizenz entnehmen Sie bitte der Lizenzinformation auf http://creativecommons.org/ licenses/by/4.0/deed.de.

\section{Literatur}

1. Achatz J, Bruckmeier K, Buch T et al (2012) Übergänge am Arbeitsmarkt und Qualität von Beschäftigung. In: Brücker H, Walwei U, Klinger S, Möller J (Hrsg) Handbuch Arbeitsmarkt 2013: Analysen, Daten, Fakten. IAB-Bibliothek, 334. wbv, Bielefeld, S 141-204 https://doi.org/10.3278/ 300776w

2. Aiken LS, West SG (1991) Multiple regression: testing and interpreting interactions. SAGE, Newbury Park (CA)

3. Atallah N, Adjibade M, Lelong H (2018) How healthy lifestyle factors at midlife relate to healthy aging. Nutrients 10:854

4. Baltes-Götz B (2013) Behandlung fehlender Werte in SPSS und Amos. ZIMK. https://www.uni-trier. de/fileadmin/urt/doku/bfw/bfw.pdf.Zugegriffen: 1. Sept. 2020

5. Borchart D, Hasselhorn HM, du Prel JB (2019) Teilnahme älterer Beschäftigter an gesundheitsfördernden und präventiven Maßnahmen. Zbl Arbeitsmed 69:261-270

6. Borchart D, Hasselhorn HM, du Prel JB (2020) Zum Gesundheitsverhalten älterer Beschäftigter - inwieweit stimmen Selbsteinschätzung und Realität überein? Präv Gesundheitsf 15:371-377

7. Dragano N, Wahrendorf M, Müller K, Lunau T (2016) Arbeit und gesundheitliche Ungleichheit: Die ungleiche Verteilung von Arbeitsbelastungen in Deutschland und Europa. Bundesgesundheitsblatt 59(2):217-227

8. Fimland MS, Vie G, Johnsen R, Nielsen TIL, Krokstad S, Bjørngaard JH (2015) Leisure-time physical activity and disability pension: 9 years follow-up of the HUNT Study, Norway. Scand J Med Sci Sports 25:e558-e565

9. Finger JD, Mensink GBM, Lange C, Manz K (2017) Gesundheitsfördernde körperliche Aktivität in der Freizeit bei Erwachsenen in Deutschland. J Health Monit 2(2):37-43

10. Fuchs J, Söhnlein D, Weber B, Weber E (2017) Ein integriertes Modell zur Schätzung von Arbeitskräfteangebot und Bevölkerung. IAB Forschungsbericht 10/2016. http://doku.iab.de/ forschungsbericht/2016/fb1016.pdf. Zugegriffen: 1. Sept. 2020

11. Fuchs J, Weber B (2020) Höhere Erwerbsquoten stoppen nicht den Rückgang des Erwerbspersonenpotenzials. Soz Fortschr 69:45-71

12. Hasselhorn HM (2020) Gesundheit und Erwerbsperspektive bei Männern und Frauen im höheren Erwerbsalter. In: Jürges H, Siegrist J, Stiehler M (Hrsg) Männer und der Übergang in die Rente Vierter Deutscher Männergesundheitsbericht der Stiftung Männergesundheit. Psychosozial-Verlag, Gießen, S91-106

13. Hasselhorn HM (2020) Wie lange wollen und können Erwerbstätige in Deutschland arbeiten? Dtsch Rentenversicher 04/2020:485-506

14. Hasselhorn HM, Ebener M(2018) The differentiated role of health foremployment participationamong older workers-a discussion based on the "lidA conceptual framework". In: Hohnerlein EM, Hennion S, Kaufmann O (Hrsg) Employment biographies and social protection in europe. Springer, Berlin, Heidelberg, New York, S 199-214

15. Hasselhorn HM, PeterR, Rauch A etal (2014) Cohort profile: the lidA cohort study - a German cohort study on work, age, health and workparticipation. Int JEpidemiol 43(6):1736-1749

16. Haukenes I, Riise T, Haug K, Farbu E, Maeland JG (2013) Smokers' increased risk for disability pension: social confounding or health-mediated effects? Gender-specific analyses of the Hordaland Health Study cohort. J Epidemiol Community Health 67:758-764

17. Haukenes I, Mykletun A, Knudsen AK, Hansen HT, Maeland JG (2011) Disability pension by occupational class - the impact of work-related factors: the Hordaland Health Study Cohort. BMC Public Health 11:406

18. Krosnick JA, Malhotra N, Mo CH et al (2019) Correction: Perceptions of health risks of cigarette smoking: a new measure reveals widespread misunderstanding. PLoS ONE14(2):e212705

19. Krug S, Jordan S, Mensink G, Müters S, Finger J, Lampert T (2013) Körperliche Aktivität - Ergebnisse der Studie zur Gesundheit Erwachsener in Deutschland (DEGS1). Bundesgesundheitsblatt 56:765-771

20. Lallukka T, Rahkonen O, Lahelma E, Lathi J (2015) Joint associations of smoking and physical activity with disability retirement: a register-linked cohort study. BMJOpen 5:e6988

21. Leinonen T, Martikainen P, Lahelma E (2012) Interrelationships between education, occupational social class, and income as determinants of disability retirement. Scand J Public Health 40(2):157-166

22. Liebermann SC, Müller A, Weigl M, Wegge J (2015) Antecedents of the expectation of remaining in nursing until retirement age. J Adv Nurs 71(7):1624-1638

23. Nilsson K, Hydbom AR, Rylander L (2011) Factors influencing the decision to extend working life or retire. Scand JWork Environ Health 37(6):473-480
24. Nübling $M$, Andersen $H H$, Mühlbacher $A$ (2006) Entwicklung eines Verfahrens zur Berechnung der körperlichen und psychischen Summenskalen auf Basis der SOEP-Version des SF12 (Algorithmus). DIW, Berlin. https://www.econstor.eu/handle/ 10419/129225.Zugegriffen: 1.Sept. 2020

25. Oude Hengel KM, Blatter BM, Geuskens GA, Koppes LLJ, Bongers PM (2012) Factors associated with the ability and willingness to continue working until the age of 65 in construction workers. Int Arch Occup Environ Health 85(7):783-790

26. Pate RR, Pratt M, Blair SN et al (1995) Physical activity and public health. J Am Med Assoc 273:402-407

27. Robert Koch-Institut (Hrsg) (2014) Gesundheitliche Lage der Männer in Deutschland. S 187-199. https://www.rki.de/DE/Content/ Gesundheitsmonitoring/Gesundheitsberichter stattung/GBEDownloadsB/maennergesundhe it.pdf?_blob=publicationFile. Zugegriffen: 1 . Sept. 2020

28. Robert Koch-Institut (Hrsg) (2017) Gesundheitliche Ungleichheit in verschiedenen Lebensphasen. Gesundheitsberichterstattung des Bundes. RKI, Destatis, Berlin, S49-72

29. Robroek SJ, Schuring M, Croezen S, Stattin M, Burdorf A (2013) Poor health, unhealthy behaviors, and unfavorable work characteristics influence pathways of exit from paid employment among older workers in Europe: a four year follow-up study. Scand JWork Environ Health 39:125-133

30. Scharn M, Sewdas R, Boot CRL, Huisman M, Lindeboom M, van der BeekAJ (2018) Domains and determinants of retirement timing: a systematic review of longitudinal studies. BMC Public Health 18:1083

31. Schienkiewitz A, Mensink GBM, Kuhnert R, Lange C (2017) Übergewicht und Adipositas bei Erwachsenen in Deutschland. J Health Monit 2(2):21-27

32. Schröder $H$, Kersting A, Gilberg $R$, Steinwede $J$ (2013) Methodenbericht zur Haupterhebung lidA - leben in der Arbeit. FDZ-Methodenreport 01/2013. Institut für Arbeitsmarkt- und Berufsforschung. http://doku.iab.de/fdz/reporte/2013/ MR_01-13.pdf.Zugegriffen: 1.Sept. 2020

33. Scott KA, Liao Q, Fisher GG, Stallones L, DiGuiseppi C, Tompa E (2018) Early labor force exit subsequent to permanently impairing occupational injury or illness among workers 50-64 years of age. Am JInd Med 61 (4):317-325

34. Siegrist J, Wege N, Pühlhofer F, Wahrendorf M (2009) A short generic measure of work stress in the era of globalization: effort-reward imbalance. Int Arch Occup Environ Health 82(8):1005-1013

35. Södergren M, McNaughton SA, Salmon J, Ball K, CrawfordDA (2012) Associations between fruit and vegetable intake, leisure-time physical activity, sitting time and self-rated health among older adults: cross-sectional data from the WELL study. BMC Public Health 12:551

36. Stenholm S, Head J, Kivimäki M (2016) Smoking, physical inactivity and obesity as predictors of healthy and disease-free life expectancy between ages 50 and 75: a multicohort study. Int JEpidemiol 45(4):1260-1270

37. van den Berg TI, Elders LA, Burdorf A (2010) Influence of health and work on early retirement. JOccup Environ Med 52(6):576-583

38. van den Heuvel S, de Wind A (2015) Domain: Health and health-related behaviour. In: Hasselhorn HM, Apt W (Hrsg) Understanding employment participation of older workers: Creating a knowledge base for future labour market 


\section{Originalien}

challenges. Research Report. Federal Ministry of Labour and Social Affairs (BMAS) and Federal Institute for Occupational Safety and Health (BAuA). http://www.jpdemographic.eu/about/ fast-track-projects/understanding-employment. Zugegriffen:01.Sept.2020

39. van Rijn RM, Robroek SJ, Brouwer S, Burdorf A (2014) Influence of poor health on exit from paid employment: a systematic review. Occup Environ Med 71(4):295-301

40. Virtanen M, Oksanen T, Pentti J et al (2017) Occupational class and working beyond the retirement age: a cohort study. Scand J Work Environ Health 43(5):426-435

41. Wilson DM, Errasti-Ibarrondo B, Low G et al (2020) Identifying contemporary early retirement factors and strategies to encourage and enable longer working lives: A scoping review. Int J Older People Nurs 15:e12313

42. World Health Organization (WHO) (2020) Body mass index - BMl. http://www.euro.who.int. Zugegriffen: 1.Sept. 2020

43. Ybema JF, Geuskens GA, van den Heuvel $S$ et al (2014) Study on transitions in employment, ability and motivation (STREAM): the design of a fouryear longitudinal cohort study among 15,118 persons aged 45 to 64 years. Br J Med Med Res 4(6):1383-1399

44. Zeiher J, Kuntz B, Lange C (2017) Rauchen bei Erwachsenen in Deutschland. J Health Monit 2(2):59-65 